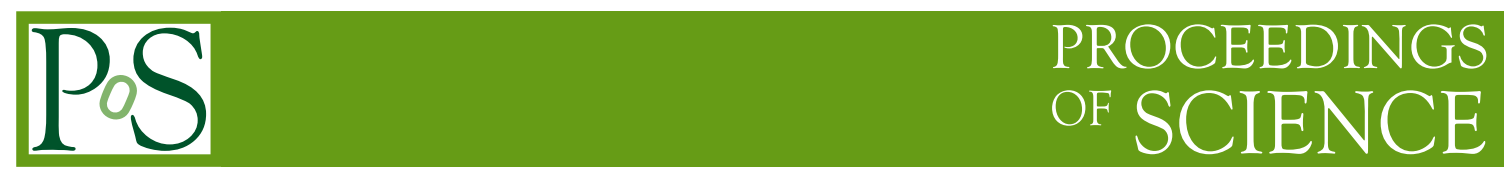

\title{
INTERPLANETARY MAGNETIC FIELD PARAMETERS AFFECTING COSMIC RAY FORBUSH DECREASE
}

\section{L. Chauhanı}

Department of Physics, Govt. Model Science College

Jabalpur, M.P., INDIA

E-mail: mansukhc24@gmail.com

\section{M.K.Richhariaz}

Department of Physics, Govt. Model Science College Jabalpur, M.P., INDIA

\section{B.K.Soni3}

Government Girls College, Bilaspur (C.G.), INDIA.

\section{Abstract}

Coronal mass ejections (CMEs) hurl huge volumes of magnetized plasma into interplanetary space often referred to as ICMEs or ejecta. They are an important component of solar wind and can cause enhanced geomagnetic activity when they interact with the Earth's magnetosphere. When the ejecta have an average speed greater than the upstream solar wind speed they create a shock. The large IMF variations due to interplanetary shocks cause depression in the cosmic ray intensity (CRI) called Forbush Decrease (FD). Large Fds caused by fast CMEs are specifically associated with energetic X-ray flares. In the present paper, the author has studied seven largest Forbush decrease events selected from Moscow Neutron Monitor Station during a period of twelve years (19962008), i.e., 23rd solar cycle. The analysis of CRI data with interplanetary magnetic field $|\mathrm{B}|$, its southward component $\mathrm{Bz}$, solar wind velocity, $\mathrm{Kp}$ and Dst indices shows that all the three phenomena- solar, interplanetary and geomagnetic are connected to FD. The relationship between interplanetary parameters and FDs is discussed in detail. Moreover the solar cycle effect is found to be slightly shifted for large FDs as the frequency of occurrence of major FD events is more in the descending phase of the solar cycle.

Key word: Cosmic ray intensity, Forbush decrease, Coronal mass ejections. 


\section{Introduction:}

Cosmic ray Forbush decreases (FDs) have been studied for more than 60 years, but even today this phenomenon is not understood fully, several questions are yet to be answered like: What determines the magnitude of FDs and their variety? How are FDs associated with disturbances of the interplanetary medium, coronal mass ejections and high speed flows of the solar wind? What relation exists between FDs and geomagnetic storms? [1]. In order to answer these questions a comprehensive study of seven exceptionally large Forbush decrease events occurred in 23 rd solar cycle has been carried out in the present paper. It is now well established that halo CMEs produce large disturbances in the solar wind and they generally reach the near earth space inside the so called high speed plasma streams [2]. These disturbances are the primary cause of geomagnetic storms [3]. On the other hand they also modulate the flux of galactic cosmic rays in the form of Forbush decrease [4]. The two-step Forbush decrease in cosmic ray records seems to originate from the structure within the shock and sheath preceding the interplanetary coronal mass ejection [5]. In this paper we investigate the affect of interplanetary magnetic field (IMF) parameters - total B \& southward component Bz along with solar wind velocity on cosmic ray intensity (CRI) during following time intervals when the FD amplitude was $>=9 \%$ recorded by Moscow neutron monitor station: 9-15 April 2001, 4-10 November 2001, 22-28 November 2001, 28 October-3 November 2003, 24-30 July 2004, 1319 May 2005 \& 9-15 September 2005. We have searched for the solar sources responsible for above events by tracking back the sun spot numbers (SSN), sun spot area, associated CME, solar flare and their location on solar disk, one-two days before the onset of FD. The two-step Forbush decrease in cosmic ray records seems to originate from the structure within the shock and sheath preceding the interplanetary coronal mass ejection [5].

In this paper we investigate the affect of interplanetary magnetic field (IMF) parameters - total B \& southward component $\mathrm{Bz}$ along with solar wind velocity on cosmic ray intensity (CRI) during following time intervals when the FD amplitude was $>=9 \%$ recorded by Moscow neutron monitor station: 9-15 April 2001, 4-10 November 2001, 22-28 November 2001, 28 October-3 November 2003, 24-30 July 2004, 13-19 May 2005 \& 9-15 September 2005. We have searched for the solar sources responsible for above events by tracking back the sun spot numbers ( $\mathrm{SSN}$ ), sun spot area, associated CME, solar flare and their location on solar disk, one-two days before the 
onset of FD. The arrival of the interplanetary shock waves at the Earth, i.e., fast forward shock, was identified by the sudden increase of the IMF intensity and the SWP bulk speed in the near-Earth space solar wind data, and as the time of the storm sudden commencement (SSC). The intense geomagnetic storms and large FDs have common origin and are observed simultaneously; the geomagnetic activity and $\mathrm{CR}$ modulation rise with increasing IMF intensity and SW velocity [1], hence to find the relation of FD with storm the variation in Dst and $\mathrm{Kp}$ indices is analyzed during the main phase of each FD event. This analysis has space weather applications too, being studied by many scientists [6,7]. Finally, the reason for occurrence of very large FD events in the descending phase of last solar cycle is reviewed.

\section{IData used}

The data sources used in our analysis are:

Hourly averaged cosmic ray variation in percent have been taken from real time database of Moscow neutron monitor station (cut off rigidity $=2.43 \mathrm{GV}, 55.47 \mathrm{~N}, 37.32 \mathrm{E}$ ) (http://cr0.izmiran.rssi.ru/mosc/main.htm).

Hourly averaged IMF B, Bz, Solar wind plasma speed, Dst \& Kp indices are provided by OMNI data (http://omniweb.gsfc.nasa.gov/).

SSN, sun spot area and geomagnetic indices are obtained from (http://www.swpc.noaa.gov/ftpdir/).

Solar flare and SSC data are sourced from (ftp://ftp.ngdc.noaa.gov/)

$=\mathrm{CME}$ data is archived from the on line LASCO list (http://cdaw.gsfc.nasa.gov/CME_list)

Interplanetary shock, ICME and magnetic cloud data are from ACE list of disturbances and transients

(http://wwwssg.sr.unh.edu/mag/ace/ACElists/).

Geomagnetic storm scale is according to the (http://www. swpc.noaa.gov/NOAA scales).

\subsection{Data Analysis}

\section{(i) FD event of 11 April, 2001}

This was the first large FD $(\sim 11 \%)$ event of maximum phase of $23_{\text {rd }}$ solar cycle. It originated from AR 9415 (S23W09) which unleashed X-ray flare of class X2.3 on 10 April 2001 at 0506 UT. Following the flare, a halo CME was observed by LASCO/SOHO at 0530 UT with a linear speed of $2411 \mathrm{~km} / \mathrm{s}$. The active region had a complex delta class magnetic field with SSN equals 170 and SSN area equal to 930 msh (millionth of solar hemisphere). On 11 April 2001, the fast CME drove an interplanetary shock, observed by ACE (L1 point, $240 \mathrm{RE}$ ) at 1314 UT, featured by 
sudden jump in solar wind proton speed and high IMF magnitudes $\triangle \mathrm{B}$ $\triangle \& \mathrm{Bz}$ as shown in Figure 1.

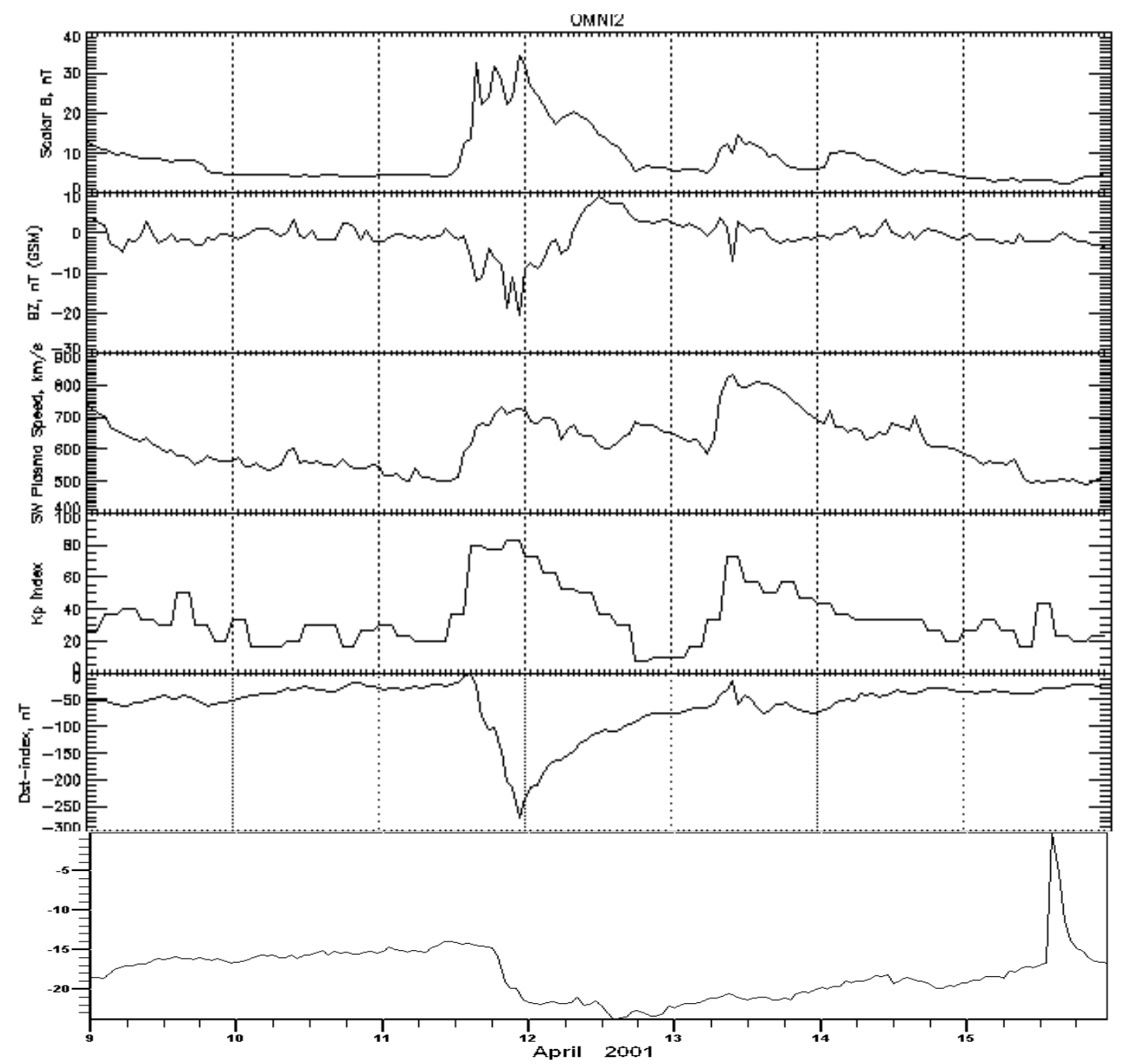

Figure 1: (from top to bottom) IMF $\mid$ B $\mid$, its southward component Bz, solar wind elocity, Dst \& Kp indices, and CR variation (all hourly averaged) plots from 9 to 15 April, 2001 at 1AU. Vertical line marks the arrival of IP shock wave at the Earth.

From figure 1, it is noticed that SW velocity jumped to $730 \mathrm{~km} / \mathrm{s}$, $\triangle \mathrm{B} \triangle$ increased to $34 \mathrm{nT}$ and $\mathrm{Bz}$ value fallen to $-20 \mathrm{nT}$. Thus due to ICME impacting on slow solar wind there was a sheath upstream of ICME led by a fast forward shock causing FD at 1302 UT on 11 April 2001. The large IMF variations in this sheath sustained the depression in the CRI during FD. They scattered the galactic cosmic rays and thus swept away the cosmic ray particles. The increase in solar wind dynamic pressure produced magnetopause compression marked by storm sudden commencement (SSC) at 1343 UT approximately after 30 minutes of the arrival of shock. 
Large fraction of solar wind energy input to the magnetosphere under the favorable $\mathrm{Bz}$ conditions contributed to the large ring current development and intense storm was observed. The Dst dipped to $-270 \mathrm{nT}$ and planetary Kp reached to a value equal to 8 (G4) on 11 April 2001.

\section{(ii)FD event of 6 November, 2001}

An energetic solar phenomena was observed in AR 9684 (N06W18) on 4th November 2001. The SSN was 186 and SSN area grew up to 1820 msh. It sparked a solar flare, X1.0 at 1603 UT and hurled halo CME (start time 1635 UT) on same day with speed of $1810 \mathrm{~km} / \mathrm{s}$.

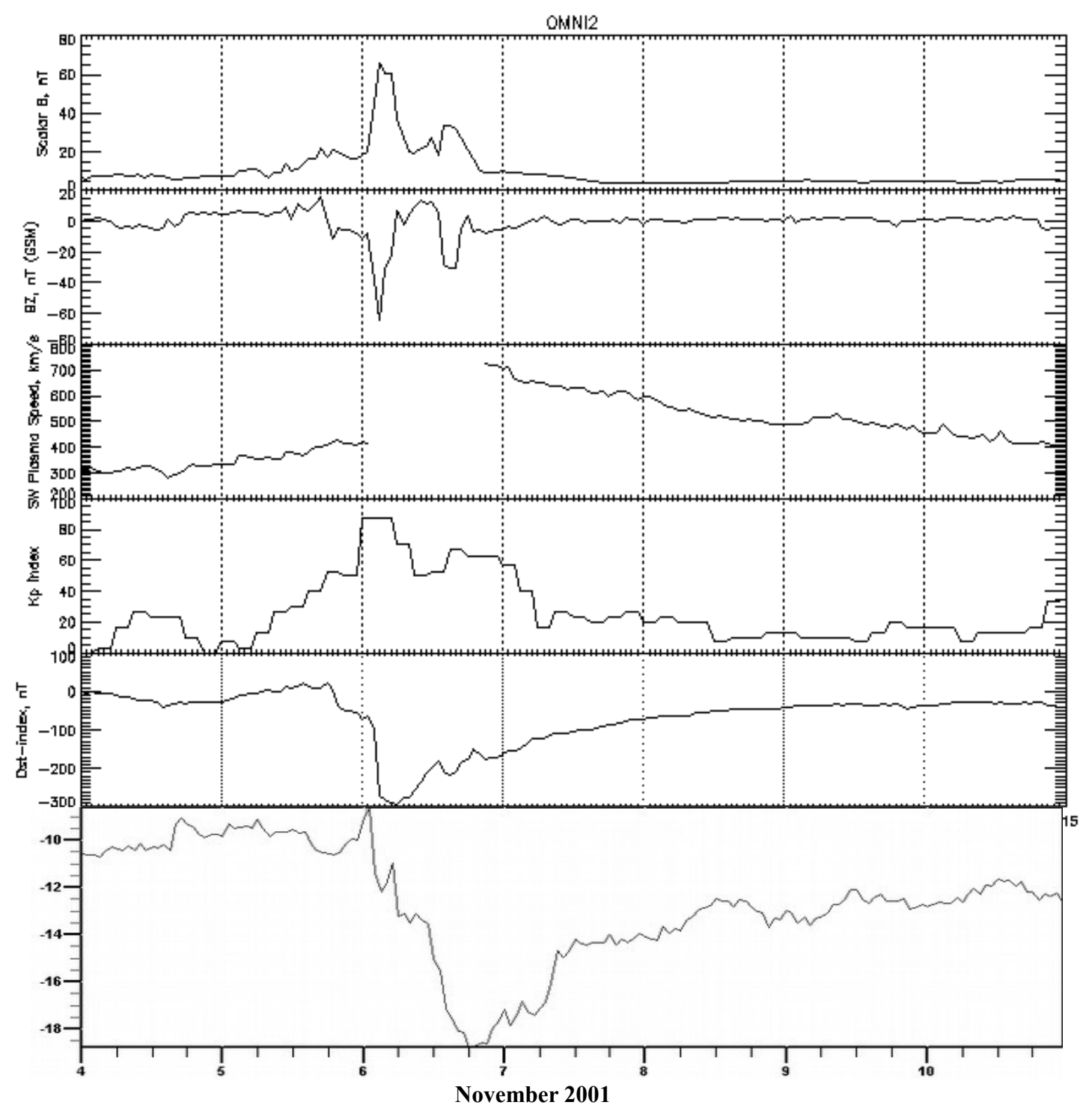

Figure 2: Summary plot as in figure 1 for the interval of 4-10 November, 2001 
The disturbances reached earth and generated shock. The shock was observed at 0125 UT, 17 minutes before the start time of ICME i.e. $0152 \mathrm{UT}$. The onset of FD was at 0115 UT which is 10 minutes earlier than the arrival of shock. An intensive FD with magnitude 10\% was recorded by majority of neutron monitors. It is evident from the figure 2 that the passage of IP shock was marked by an extraordinary large increase in magnetic field strength, $\triangle \mathrm{B} \triangle$ was increased to $65 \mathrm{nT}$ and $\mathrm{Bz}$ to $-65 \mathrm{nT}$. The solar wind velocity rose suddenly to $790 \mathrm{~km} / \mathrm{sec}$. These large values imply that ICME interacted with the Earth's magnetosphere to a large extent. Shock and ejecta produced two steps FD with no magnetic cloud structure associated with it. The event was accompanied by an intense geomagnetic storm of Dst $\sim-300 \mathrm{nT}$ and $\mathrm{Kp}=8$. The storm was categorized as G4 (severe) by NOAA scale. IMF $\triangle \mathrm{B} \triangle$ remained high for a few hours, indicating a turbulent sheath region. Hence, CRI also remained depressed for a few hours during the passage of high field. But the ppearance of SSC neither coincided with FD nor with shock. It started at 0648UT on 6 November 2001, five hours later the occurrence of FD.

\section{(iii)FI event of 24 November, 2001}

On 22nd November the SSN and SS area were 143 and $1310 \mathrm{msh}$, respectively. AR 9704 erupted a halo CME at 2330 UT on 22 nd November 2001. Simultaneously, an M9.9 class solar flare was also emitted at 2232 UT. The CME traveled with a speed of $1437 \mathrm{~km} / \mathrm{s}$ towards Earth.

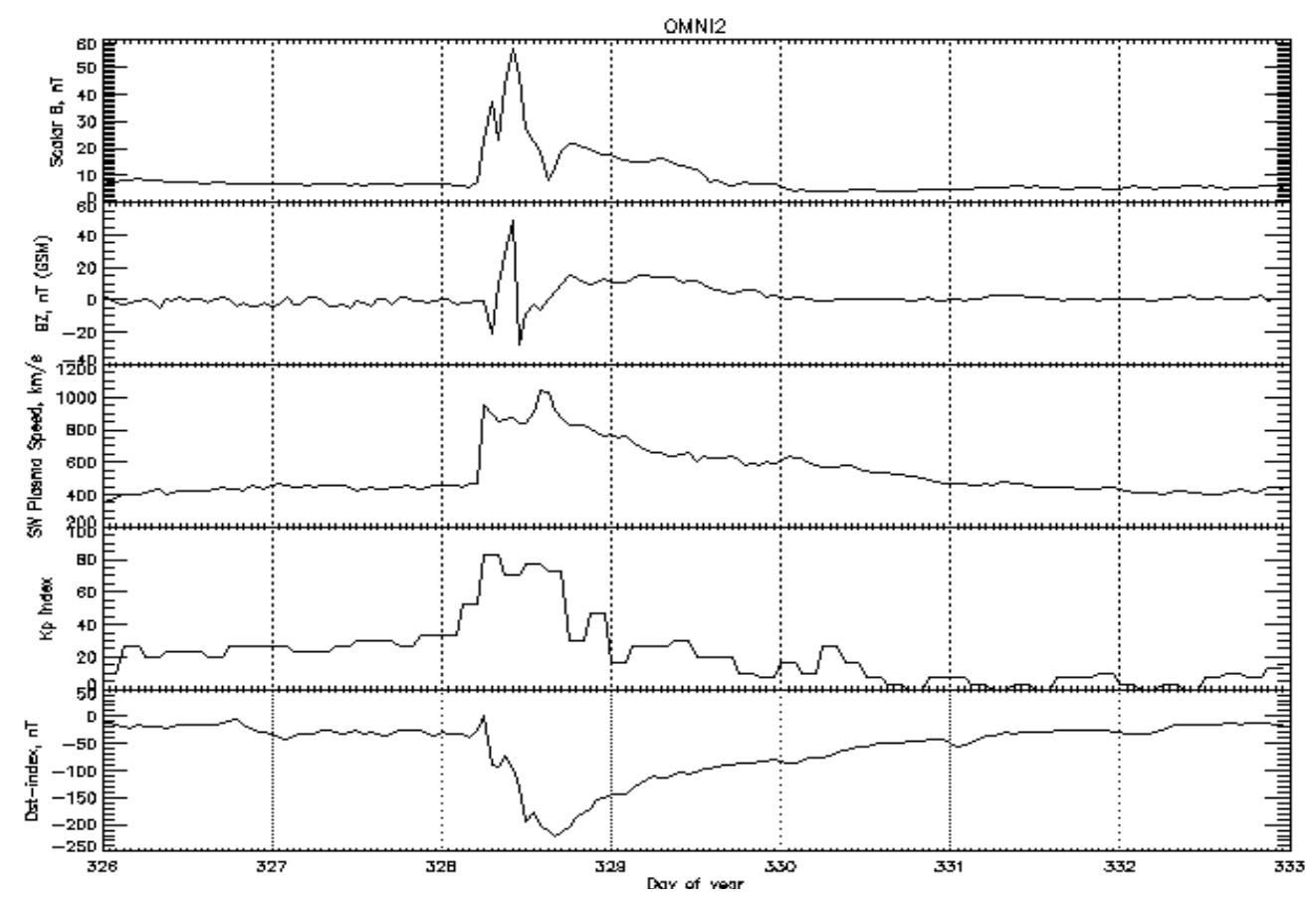

Figure 3: Summary plot as in figure 1 for the interval 22-28 November, 2001. 
Energetic particles accelerated by the flare and associated CME, caused lot of disturbances on 24 November 2001 at a distance 1AU from Earth. The expanding ICME swept past our planet at 0656 UT along with magnetic cloud on 24 November. It was preceded by a shock at 0538 UT with sudden decrease in cosmic ray density i.e. FD 9\% at 0533 UT. The SSC appeared with above events at 0554 UT. This implies that the shock, SSC \& FD occurred simultaneously. The solar wind speed got highly elevated with a maximum value of $1050 \mathrm{~km} / \mathrm{s}$. IMF total magnetic field reached to a high value of $58 \mathrm{nT}$ with its southward component $\mathrm{Bz}$ to $-25 \mathrm{nT}$. All these conditions favoured an extreme storm with minimum Dst $\sim-220 \mathrm{nT}$ and $\mathrm{Kp}$ index $\sim 9$ (G5). The variation graphs in the interval between 22 November to 28 November 2001 for interplanetary, geomagnetic and CRI data can be viewed in figure 3. The time profiles show the dependence of CRI on interplanetary magnetic field conditions. Large FDs are caused by big increase in IMF is noticed by above analysis. The Dst index started decreasing after few hours after the onset of FD and had same pattern as CRI variation indicating that the phenomenon may have same origin. There were three forward shocks reported by MAG on 24 November, at 0538UT, 0800UT and at 0900UT in which second one had $400 \%$ (30nT) jump in $\triangle \mathrm{B} \triangle$ and third one had $50 \%$ (25nT) jump in $\triangle \mathrm{B}$ $\triangle$. Since magnetic cloud structure was also found during the event period, the galactic cosmic rays are also scattered away from the high magnetic field pressure in the magnetic cloud.

\section{(iv) FD event of 29 Detober, 2003 (Halloween event)}

On 26 October 2003 two giant spots appeared on the face of the sun. The sunspot group rapidly increased in size and early hours of 28 October 2003 it occupied an area of $4520 \mathrm{msh}$ with SSN equal to 230 . On same day a powerful flare of X17.2 class was sparked from AR 10486 at 0951 UT (S16E08). The explosion hurled a halo coronal mass ejection at $1130 \mathrm{UT}$ traveling with speed of $2459 \mathrm{~km} / \mathrm{s}$. The transit time of CME was $\sim 19$ has found to be consistent with as reported by [13]. This event is studied by [7] in detail. From figure 4, it is evident that the resulting ICME swept pass the Earth producing a shock ahead at 0558 UT on 29 October 2003. Then onset of ICME was started at 0611UT with no magnetic cloud associated with the event. The effect on Earth reflected by the appearance of SSC on 26 October at 0613UT with a record rise in solar wind proton speed up 1900 $\mathrm{km} / \mathrm{s}$. IMF increased up to $48 \mathrm{nT}$ and its southward component went to $-28 \mathrm{nT}$, showing high magnetospheric variations. The geomagnetic activity index Dst dipped to $-350 \mathrm{nT}$ causing a severe storm $\mathrm{Kp}=9(\mathrm{G}=5)$. The 
strong depression in cosmic ray flux was registered ( 22\%) at $0600 \mathrm{UT}$ on 29 October 2003. In connection to cosmic ray flux, the neutron monitors all over the world recorded a decrease of $20-30 \%$ in their counts; the largest FD of this solar cycle.

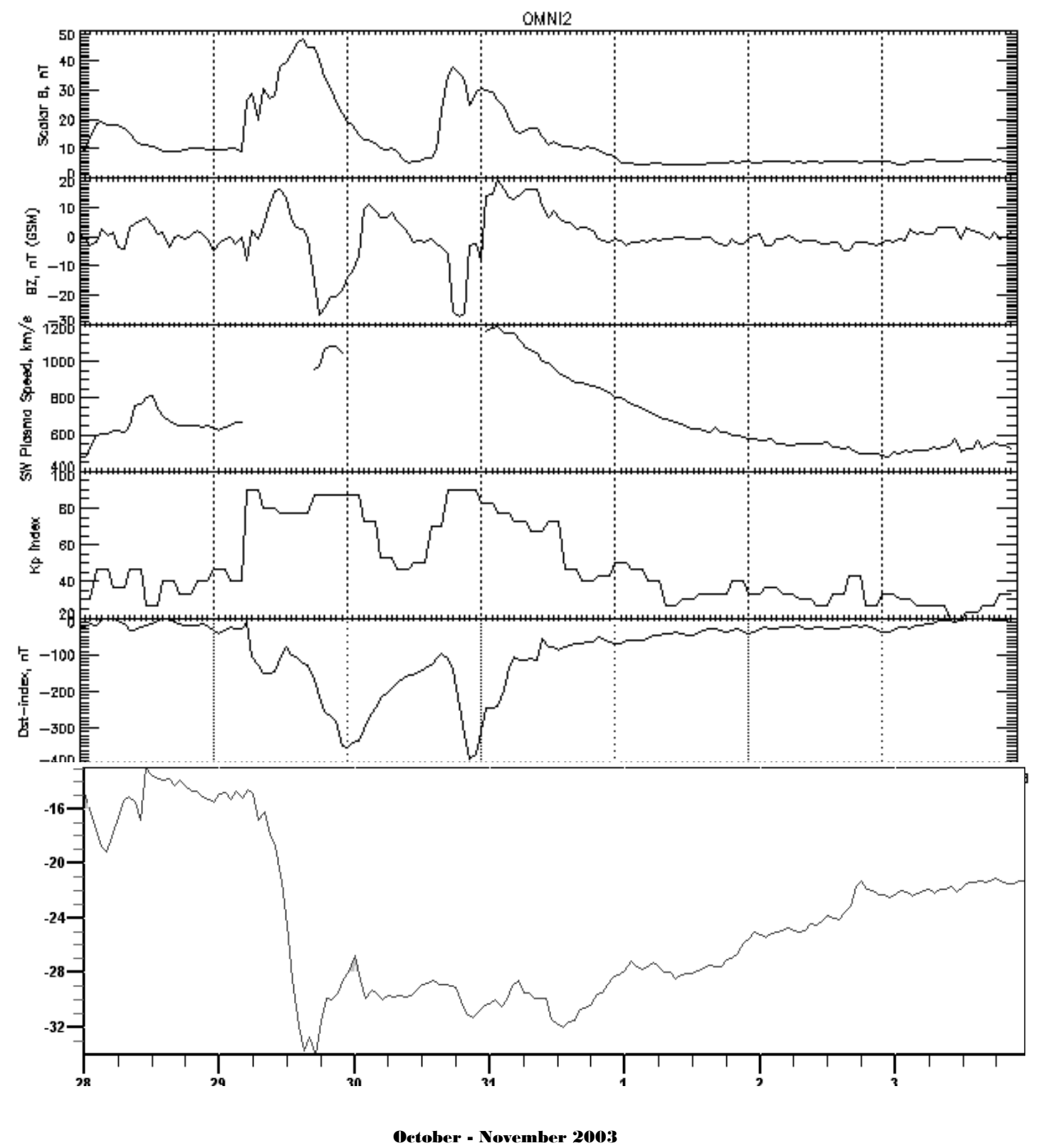

Figure 4: Summary of the data as described in figure 1 for the interval 28 October to 3 November, 2003.

One can notice that the arrival of shock coincides with the onset of FD and the peak of $\triangle B \triangle$ and maximum FD were at the same time, but the magnitude of FD can be accounted more to the large of solar wind velocity because value of $\triangle B \triangle$ maximum is less in comparison to the FD 
event of 6 November 2001. There is a time lag in the onset of Dst and onset of FD which can be used for space weather forecasting [12].

\section{(v)FI event of 26, July 2004}

A major FD event $(\sim 10 \%)$ was observed in the declining phase of 23 rd solar cycle on 26 July 2004 . The sequence of solar events related to it were: high sun spot number $\sim 130$ and raised sunspot area $\sim 1440$ msh were observed on 25 July 2004, the active region 10652 (N04W30) continued to grow since 22 nd July, became bigger than the planet Jupiter. It exploded M2.2 solar flare at 1337 UT on 25 July 2004. A halo CME followed it with starting time of 1454 UT and speed of $1333 \mathrm{~km} / \mathrm{s}$. The CME reached at Earth at 2230 in the form of ICME structure at 2249 UT and produced forward shock in succession of SSC at 2250 UT.

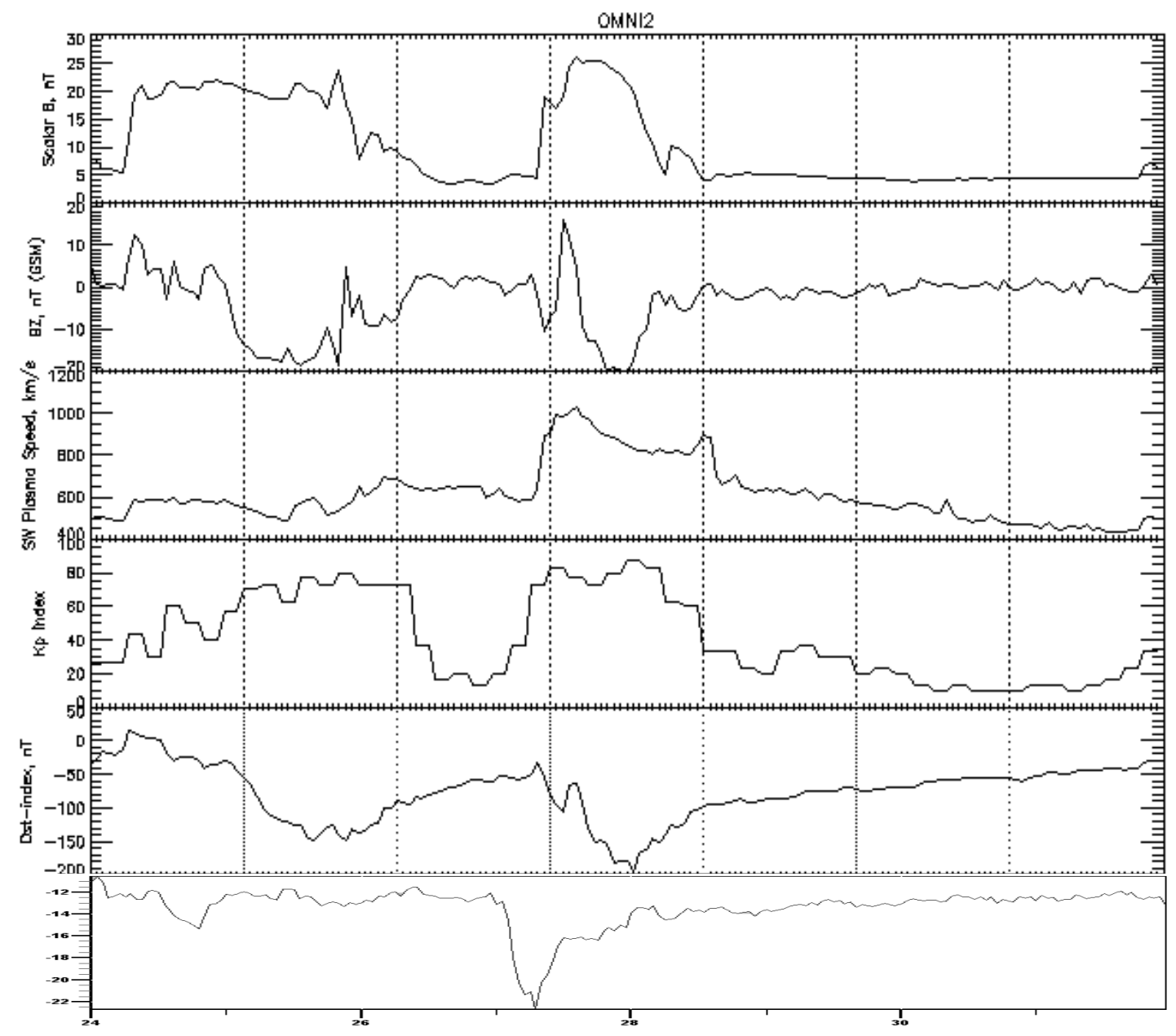

July 2004

Figure 5: Summary of the data as described in figure 1 for the interval 24-30, July 2004 
A seven day summary (from 22 to 27 July 2004) of different interplanetary (B and $\mathrm{Bz}$ ), geomagnetic ( $\mathrm{Kp}$ and $\mathrm{Dst}$ ) and cosmic ray intensity can be viewed in Figure 5. These graphs show how the interaction of intense flare and fast CME influenced magnetosphere and cosmic ray flux. The impact of ICME resulted in a sudden jump in solar wind velocity to $1020 \mathrm{~km} / \mathrm{s}$. The IMF fluctuations got enhanced; $\triangle \mathrm{B} \triangle \sim 26 \mathrm{nT}$ and $\mathrm{Bz}$ $\sim-20 \mathrm{nT}$, larger then average value. All these conditions produced a shielding of cosmic rays in the way to Earth i.e. an FD event occurred at 2228 UT on 26 July 2004. The disturbance in Earth's magnetic field could be assessed by fall in Dst index. It went to $-200 \mathrm{nT}$ with $\max \mathrm{Kp}=9$. The depression in CRI appeared to be caused by enhanced convection and particle scattering by solar wind. There was no magnetic cloud observed on 26 July 2004.

\section{(vi)FD event of 15 May 2005}

In spite of declining phase the sun became very active in May 2005. SSN became 100 on 13th May and sunspot area was measured as $1280 \mathrm{msh}$. Sunspot 10758 (N12E11) unleashed a strong M8.0 class solar flare at 1613UT on 13th May 2005. The blast produced a CME at 1712UT. Its speed was $1698 \mathrm{~km} / \mathrm{s}$ and in about 33 hours it hit Earth's magnetic field.

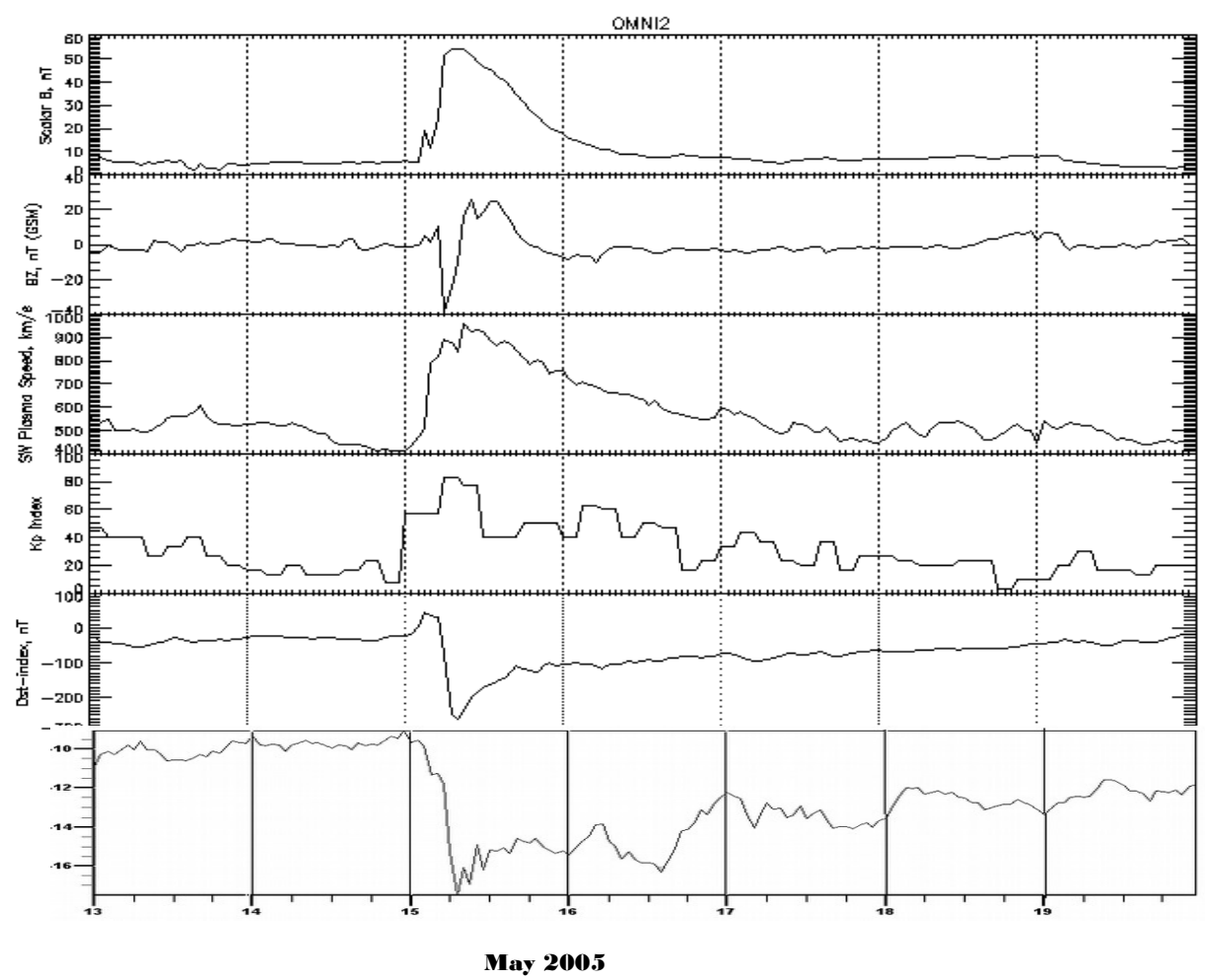

Figure 6: Summary of the data as described in figure 1 for the interval 13-19 May, 2005 
As a consequence a fast forward shock was observed at 0205 UT on 15 May ahead of ICME at 0238 UT with magnetic cloud structure. The impact caused SSC at 0239 UT and decrease in counts of neutron i.e. an FD was registered on 15 May 2005 at 0218 UT with amplitude $\sim 11.0 \%$. The graphs in figure 6 help us to analyse the overall affect of solar events at $1 \mathrm{AU}$ on Earth's magnetosphere as well as cosmic rays reaching at ground. By this the correlation of FD with all the parameters can be evaluated. One notes that large FDs are caused by high IMF structures (compared to the size of Earth) convicted by the solar wind past the Earth, the interplanetary remnants of the fast coronal mass ejection (ICMEs). The amplitude of an FD is largest when the Earth is located deep inside an ICME where GCR density is lower [10]. The passage of ICME resulted in increase of solar wind velocity to a high value of $960 \mathrm{~km} / \mathrm{sec}$. The interplanetary magnetic field also became strong and turbulent with its $\triangle \mathrm{B} \triangle, \sim 55 \mathrm{nT}$ and $\mathrm{Bz} \sim$ $-40 \mathrm{nT}$. The large southward IMF $\mathrm{Bz}$ triggered an extreme geomagnetic storm with deepest Dst $\sim-260 \mathrm{nT}$ and maximum Kp $\sim 9$.

\section{(vii)FD event of 11 September, 2005}

Though the SSN on 9 September 2005 was only 59 but the area was large $\sim 1450 \mathrm{msh}$. The active sunspot 10808 (S12E67) generated a strong solar flare (X6.2) on 9 September 2003 at 1913UT and hurled halo CME at 1948 UT, the speed of CME was very fast $\sim 2257 \mathrm{~km} / \mathrm{s}$. An ICME was observed at 0114 UT without any magnetic cloud. It followed a large shock at 0100 on 11 September at Earth, a compressed plasma and turbulent field region (shock/sheath) driven by ICME produced the decrease in cosmic ray flux due to scattering of particles. A Forbush depression of about $13 \%$ amplitude started at 0049 UT on September 11, 2003. The shock arrived at the time approximately same as the onset of FD after 29 hours of CME lift off. The interplanetary parameters raised to high values $\triangle \mathrm{B} \triangle, \sim 18 \mathrm{nT}$ and $\mathrm{Bz}$ decreased to $-8 \mathrm{nT}$ both were less in comparison to the values during previous events but the amplitude of FD is still larger here. This is contributed to the high jump in solar wind speed that had increased to 1000 $\mathrm{km} / \mathrm{s}$ so the effect of solar wind velocity is found to be more significant in case of large FDs. From figure 7 it is noticed that the interplanetary abnormalities (including Bz \& Vsw) seem to have started at about 0100UT on September 11, while abnormalities of Dst seen to have started a few hours later. The onset of FD was at 0049UT, coinciding approximately with arrival of shock. The influence on the Earth's magnetic field was also severe (Dst $\sim-150 \mathrm{nT}$ ) with $\max \mathrm{Kp}=9 \& \mathrm{G} 5$; an intense storm on NOAA scale. 


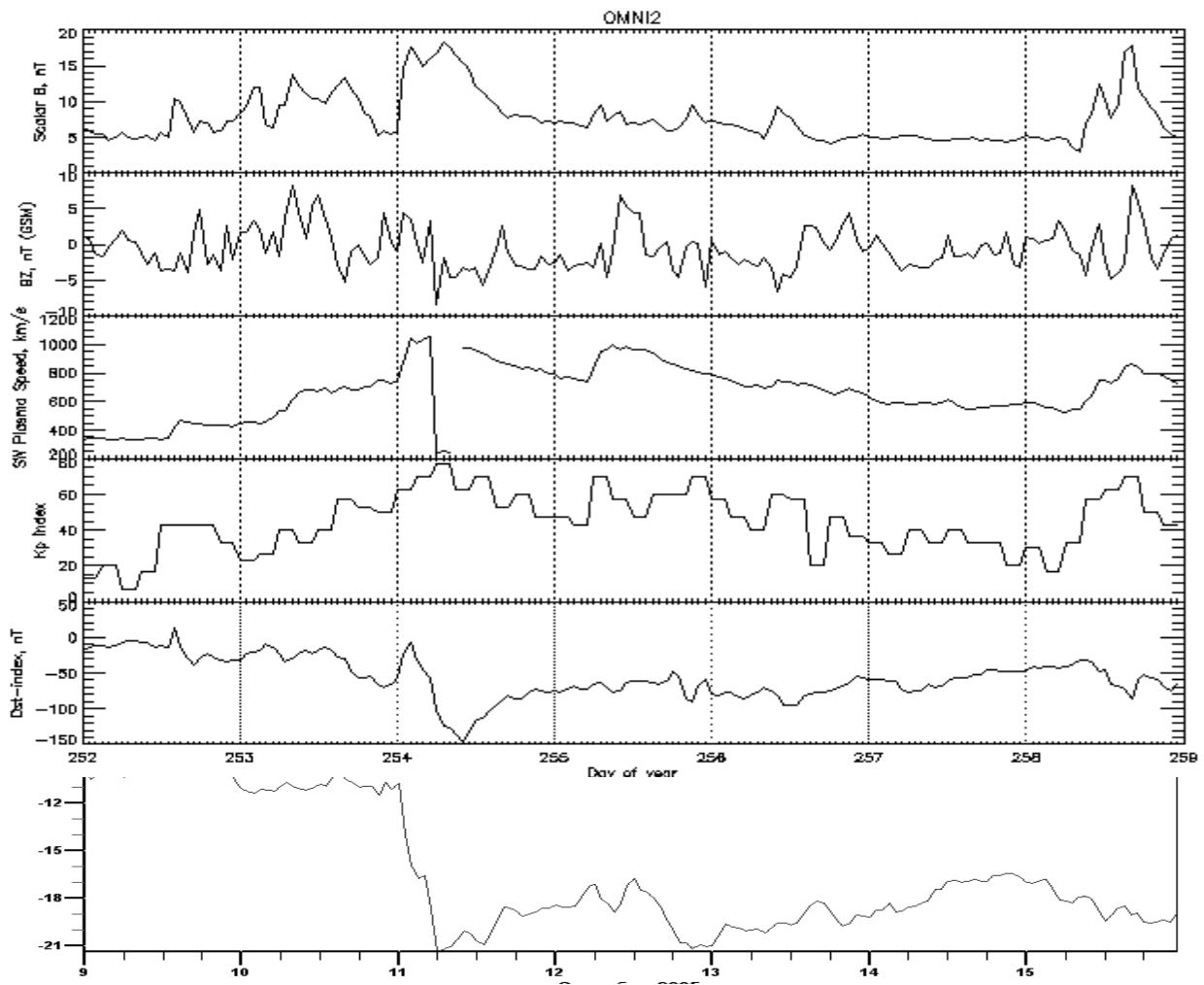

September, 2005

Figure 7: Summary of the data as described in figure 1 for the interval 9-15 Sept., 2005

\section{Discussion and Results}

In the present analysis we examined seven extreme Forbush decrease events in the period (1996-2008) in association with solar, interplanetary and geomagnetic parameters to find the causes of large depressions in cosmic ray neutron monitor counts at the Earth. It is observed that the initiation of FDs is linked to the appearance of active regions on solar disk which erupted fast CMEs associated by strong flares. The effects of interplanetary manifestation of $\mathrm{CME}$ included shocks preceded by ICME/MC, large IMF variations, sudden rise in solar wind velocity, decrease in cosmic ray flux and enhanced geomagnetic activity. This paper summarizes the extreme solar activity and its implications via Forbush decrease events during the maximum and declining phase of the solar cycle 23: 11 April 2001(AR 415), 6 \& 24 November 2001 (AR $684 \&$ 704), 28 October 2003 (AR 486), July 2004 (AR 652), May 2005 (AR 759), and September 2005 (AR 808). We have compiled and compared the properties of FDs which has the potential of yielding a lot of information for space weather applications. Understanding the behavior of such super active 
regions is very important for prediction purposes [7]. On comparing these events the following inferences can be drawn -

1. All the active regions responsible for large FDs were located close to the disk center. CMEs originating from close to the disk center (most of which become front-side halos) directly impact Earth and produce geomagnetic storms provided their magnetic field has a southward component, $\mathrm{Bz}$ [8].

2. The magnitude of FD does not depend on SSN though the sun spot area is above the median value $(800 \mathrm{msh})$ for all events. It is noted that the sun spot number and sun spot area is highest for 29 October 2003 event which is the largest event of the solar cycle 23 .

3. For producing large FDs, CME should be Earth directed and should be able to drive shocks. The linear speed of CME associated with all Fds was found to be greater than $1300 \mathrm{~km} / \mathrm{s}$ with maximum value of 2459 $\mathrm{km} / \mathrm{s}$. Since all events are associated with fast halo CMEs and intense solar flares, fast CMEs accompanied by strong solar flares are the main cause of above events. The magnitude of FD is well dependent on speed of CME and its angular width.

4. All FDs discussed have fast forward shocks ahead of ICMEs associated with them, which imply interplanetary shocks are necessary to scatter GCR away from the Earth. The generations of shocks upstream of fast ICMEs which may be important accelerators of energetic particles produce short-term (Forbush) decreases in the galactic comic ray intensity [9]. Out of seven events, three events are associated with magnetic clouds excluding largest event, implying that magnetic cloud structure is not the main cause of FD.

5. The interaction of ICME with solar wind is reflected in abrupt jump in Vsw (maximum value is $1900 \mathrm{~km} / \mathrm{sec}$ on 29 October 2003) observed for each event. The magnitude of large FD is related to solar wind velocity is another outcome of this investigation. According to [1] close relation was found between an average magnitude of the Forbush Effect and the product of the maximal solar wind velocity and the maximal IMF intensity (HmaxVmax). This fact will be analysed in future with wider range of data.

6. The disturbances in magnetosphere increase the value of interplanetary magnetic field intensity. The IMF magnitude and direction are the most important parameters controlling the magnitude of magnetic storms. But the magnitude of FD is correlated to high values of $\triangle B \triangle$, and less related to its southward component Bz. It is not clear that why $\triangle \mathrm{B} \triangle$ is maximum $\sim 65 \mathrm{nT}$ for 6 November 2001 event whose magnitude is $10 \%$ 
and for the largest event i.e. 28 October 2003, value of $\Delta B \Delta$ was lesser 48 nT. However, the large IMF variations in the shocked plasma scatter the galactic cosmic rays and thus sweep away the cosmic ray particles [4] is found true. Forbush effects (FEs) are usually observed simultaneously with an increase in the Interplanetary Magnetic Field (IMF) strength. As a rule, large Forbush decreases correspond to big increases in the IMF [11].

7. The Dst index is deepest $\sim-350 \mathrm{nT}$ for 29 October 2003 event and lowest $\sim-150 \mathrm{nT}$ for 11 September 2005 event. The magnitude of FD has no linear relation with Dst index, though large FDs are always associated with intense geomagnetic storms $(K=8$ or 9$)$. This seems to be useful for space weather forecasting [14].

8. The solar cycle effect is not very significant in case of very large FD events ( 9\%). It is seen that high amplitude FDs have occurred in the descending phase of solar cycle in more number: the maximum amplitude FD was found in beginning of declining phase. We can say that large Fds are most common near solar maximum but occur throughout the solar cycle. There are fewer than $10 \mathrm{Fds}$ greater than $10 \%$ per cycle and they occur around sunspot maximum but notably not in the year or so just after solar maximum [11].

We have presented a preliminary analysis of 7 Forbush decreases that were recorded during solar cycle 23. The solar origin and geospace consequences associated with the seven sets of events were well observed so we have an extremely rich data set, which has the potential of yielding a lot of information for understanding the behavior of transient decreases in cosmic ray flux and space weather applications.

\section{Ackmowledgements}

The authors are grateful to the research teams working in Moscow neutron monitor station, ACE/Wind, LASCO/SOHO, OMNI, and NOAA data centers for providing the related data. Mrs. Manjula Jain is thankful to UGC for sanctioning TRF for this research work.

\section{References}

[1] Storini, M., Galactic cosmic-ray modulation and solar-terrestrial

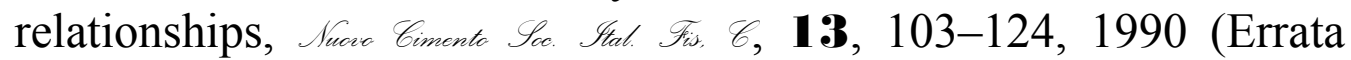

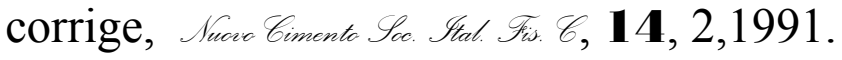

[2] Gosling, J. T., Coronal mass ejections: The link between solar and

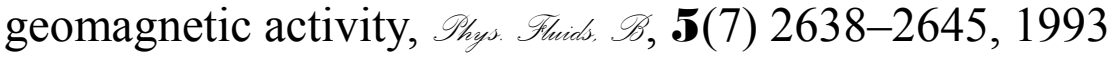

[3] Caroubalos C, P. Preka-Papadema, H. Mavromichalaki, X. Moussas, 
Papaioannou, E. Mitsakou, A. Hillaris, Space storm measurements of the July 2005 solar extreme events from the low corona to the Earth, Adv. In Space Res., 43 (2009) 600-604

[4] Ifedili, S. O., The two-step Forbush decrease: An empirical model,

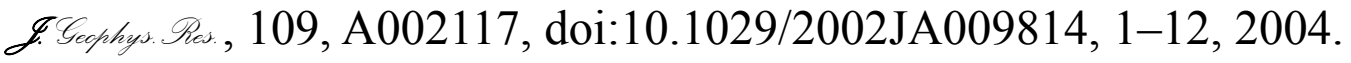

[5] Belov A. V, E. A. Eroshenko, V. A. Oleneva, A. B. Struminsky, and V. G. Yanke What determines the Magnitude of forbush decrease? Sprace Shes. Vol. 27,No. 3, pp. 625-630,2001

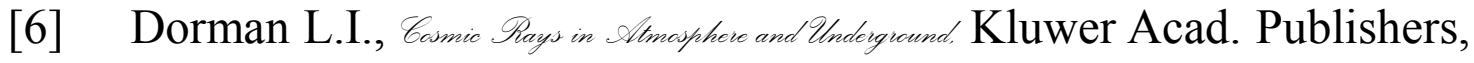
Netherlands, 2003

[7] Gopalswamy N., S. Yashiro, and S. Akiyama, Coronal mass ejections and space weather due to extreme events, ILWS WORKSHOP 2006, GOA, FEBRUARY 19-24, 2006

[8] Gopalswamy N, L. Barbieri, E. W. Cliver, G. Lu, S. P. Plunkett, and R. M. Skoug, Introduction to violent Sun-Earth connection events of October-November 2003 JOURNAL OF GEOPHYSICAL RESEARCH, VOL. 110,doi: 10.1029/2005JA011268, 2005

[9] Richardson Ian \& Hilary Cane, Interplanetary Coronal Mass Ejections During 1996-2007, 30th ICRC, Merida, Mexico, 3-11 July, 2007

[10] Ahluwalia H.S., Y. Kamide, Gnevyshev gap, Forbush decreases, ICMEs and solar wind electric field relationships, Adv. Space Res., 35 (2005) 2119-2123.

[11] Cane H.V., Coronal Mass Ejections and Forbush Decreases, Space Sci. Rev., 93, 55-77, 2000

[12] Storini, M. (2005), Transient phenomena in the heliosphere and terrestrial effects, AIP Conf. Proc., 516,120-139.

[13] Schwenn R. Dal Lago A, Huttunen E \& Ganzalez W D, Ann Geophys (France), 23 (2005) 1033

[14] Karel Kudela, Cosmic Rays and Space Weather: Direct and Indirect

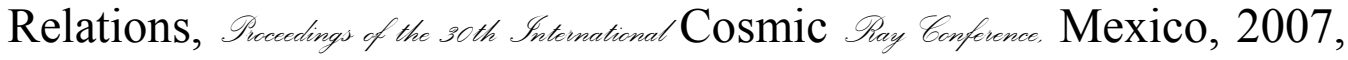
Vol. 6, pages 195-208, 10. 